\title{
A Parallel Manipulator with Planar Configurable Platform and Three End-Effectors
}

\author{
Jaime Gallardo-Alvarado (i) and Jesus H. Tinajero-Campos \\ Department of Mechanical Engineering, Instituto Tecnológico de Celaya, TecNM, Celaya 38010, GTO, Mexico \\ Correspondence should be addressed to Jaime Gallardo-Alvarado; jaime.gallardo@itcelaya.edu.mx
}

Received 15 July 2019; Revised 27 August 2019; Accepted 7 September 2019; Published 25 September 2019

Academic Editor: Gustavo Scaglia

Copyright (c) 2019 Jaime Gallardo-Alvarado and Jesus H. Tinajero-Campos. This is an open access article distributed under the Creative Commons Attribution License, which permits unrestricted use, distribution, and reproduction in any medium, provided the original work is properly cited.

\begin{abstract}
This work reports on the kinematic analysis of a planar parallel manipulator endowed with a configurable platform assembled with six terminal links serially connected by means of revolute joints. This topology allows the robot manipulator to dispose of three relative degrees of freedom owing to the mobility of an internal closed-loop chain. Therefore, the proposed robot manipulator can admit three end-effectors. The forward displacement analysis of the configurable planar parallel manipulator is easily achieved based on unknown coordinates denoting the pose of each terminal link. Thereafter, the analysis leads to twelve quadratic equations which are numerically solved by means of the Newton homotopy method. Furthermore, a closed-form solution is available for the inverse position analysis. On the contrary, the instantaneous kinematics of the robot manipulator is investigated by means of the theory of screws. Numerical examples are included with the purpose to illustrate the method of kinematic analysis.
\end{abstract}

\section{Introduction}

A typical parallel manipulator (PM) consists of a rigid moving platform connected to a fixed platform by means of two or more kinematic chains. Drawbacks and advantages of PMs have been widely discussed in the literature, and as a result of this fruitful interchange of ideas, PMs have found practical applications for industry, space, and medical science like flight and automobile motion simulators, highspeed multiaxis machining, assembly, medical parallel robot, and in general pick-and-place operation. However, in spite of the appreciable advance reached in the field, topics like reduced workspace and recurrent presence of singularities still being the motive of ardent communications in the kinematician community concerned with the performance of PMs. These drawbacks may be alleviated replacing the rigid movable platform by a configurable platform. It was thus that the term "Parallel Robots with Configurable Platforms" (PRCP) was coined in kinematics as an option to overcome the poor performance of PMs [1]. The idea to develop robot manipulators with the ability to configure an internal closed-loop chain substituting the typical rigid moving platform is without doubt a genuine concept that can encourage the creativity of the next generation of robots with parallel kinematic structures. At this point, it is worth clarifying that the concept of a configurable platform differs from the concepts of reconfigurable parallel manipulator $[2,3]$ and foldable platform [4]; e.g., a foldable platform must be considered as a collapsible platform instead of an internal closed-loop chain. Therefore, the history of PMs with configurable platforms is relatively short. In fact, to the best knowledge of the authors, the concept of a configurable platform dates back to the first years of the twenty-first century when Yi et al. [5] presented a planar PM endowed with a four-bar mechanism was introduced as a configurable platform for grasping irregular objects. Few years later, in Mohamed and Gosselin' study [6], the concept of a configurable platform was extended from planar to spatial PMs. Recently, the instantaneous kinematics and static analysis of limited-dof PRCP mechanisms were approached by Hoevenaars et al. [7]. Finally, in [1], the fundamental aspects of PRCP mechanisms enhancing their attributes such as the high capacity of grasping irregular objects via multiple 
contact points keeping the well-known advantages of classical PMs were well discussed.

In short, while the concept of a configurable robot has been widely employed for serial and conventional parallel manipulators as well as for modular robots, see, for instance, references $[3,8-18]$, the idea of a parallel manipulator with a configurable platform is a relatively new concept introduced in the field of robotics. Following that fashion, in this work, a planar parallel manipulator with a configurable platform is proposed. The displacement analysis of the robot is achieved by formulating closure equations based on the signed distance between selected points of the parallel manipulator. Thereafter, while for the forward displacement analysis, the Newton homotopy method is applied to solve twelve quadratic equations; for the inverse displacement analysis, it is possible to obtain a closed-form solution. Afterwards, the instantaneous kinematics of the proposed PRCP mechanism is carried out by means of the theory of screws. The versatility of the robot is such that different operation modes are available according to the desired number of end-effectors. Numerical examples are provided with the purpose to illustrate the method of kinematic analysis.

The main contributions of the paper may be summarized as follows: (i) the topology of a kinematically redundant fully parallel planar manipulator equipped with a configurable platform is introduced, (ii) the availability of three internal freedoms of the robot manipulator allows to dispose of two or three end-effectors, (iii) simultaneous tasks may be achieved by the robot due to the existence of multiple end-effectors, (iv) the method introduced to solve the finite kinematics of the robot allows to find all the real solutions of the inverseforward position analyses, and (v) the instantaneous kinematics of the robot is performed by resorting to the theory of screws enhancing the corresponding Jacobian matrices.

\section{Preliminary Concepts}

2.1. Basics of Screw Algebra. The mathematical resource chosen to approach the infinitesimal kinematics of the robot at hand is the screw algebra which is isomorphic to the Lie algebra se (3). As a consideration for readers unfamiliar with the issue, in this subsection, some foundations of screw algebra are briefly explained. For details of this subsection, readers are referred to [19].

In Plücker coordinates, a screw is a line vector $\$$ associated with a point $O$ of the physical space that may be written as a six-dimensional vector as follows:

$$
\$=\left(\hat{\mathbf{s}}, \mathbf{s}_{O}\right)=(\hat{\mathbf{s}}, \mathbf{r} \times \hat{\mathbf{s}}+h \mathbf{s})
$$

where $\hat{\mathbf{s}}$ denotes the direction of the line, $\mathbf{r}$ is a vector pointed from an arbitrary point on the line to point $O$, and $h$ is the pitch of the screw. The concatenated vectors $\hat{\mathbf{s}}$ and $\mathbf{s}_{O}$ are known as the primal and dual parts of $\$$, respectively, i.e., $\$=(p(\$)$ and $d(\$))=\left(\hat{\mathbf{s}}, \mathbf{s}_{O}\right)$. The screws may be associated with kinematic pairs, e.g., if the pitch $h$ is null, then the screw is related with a revolute joint yielding $\$=(\hat{\mathbf{s}}, \mathbf{r} \times \hat{\mathbf{s}})$. Meanwhile, if $h$ goes to infinity, then the screw is related with a prismatic joint and we have $\$=(0, \hat{\mathbf{s}})$. Any lower kinematic pair may be represented by screws; e.g., besides revolute and prismatic pairs, the cylindrical joint is the combination of a revolute joint with a prismatic joint, while the spherical joint is the combination of three revolute joints whose primal parts intersect the same point.

Let $\$_{1}=\left(\mathbf{s}_{1}, \mathbf{s}_{\mathrm{O} 1}\right), \$_{2}=\left(\hat{\mathbf{s}}_{2}, \mathbf{s}_{\mathrm{O} 2}\right)$, and $\$_{3}=\left(\hat{\mathbf{s}}_{3}, \mathbf{s}_{\mathrm{O} 3}\right)$ be three elements of se(3). The screw algebra has the following operations:

(1) Addition: $\$_{1}+\$_{2}=\left(\hat{\mathbf{s}}_{1}+\hat{\mathbf{s}}_{2}, \mathbf{s}_{\mathrm{O} 1}+\mathbf{s}_{\mathrm{O} 2}\right)$.

(2) Multiplication by a scalar: $\lambda \$_{1}=\left(\lambda \hat{\mathbf{s}}_{1}, \lambda \mathbf{s}_{\mathrm{O} 1}\right)$, where $\lambda \in \mathbb{R}$.

(3) Lie product: $\left[\begin{array}{ll}\$_{1} & \$_{2}\end{array}\right]=\left(\hat{\mathbf{s}}_{1} \times \hat{\mathbf{s}}_{2}, \hat{\mathbf{s}}_{1} \times \mathbf{s}_{\mathrm{O} 2}-\hat{\mathbf{s}}_{2} \times \mathbf{s}_{\mathrm{O} 1}\right)$. Properties of the Lie product are as follows:

Nilpotent: $\left[\begin{array}{ll}\$_{1} & \$_{1}\end{array}\right]=(0,0)$.

Distributive: $\left[\begin{array}{ll}\$_{1} & \lambda_{2} \$_{2}+\lambda_{3} \$_{3}\end{array}\right]=\lambda_{2}\left[\begin{array}{ll}\$_{1} & \$_{2}\end{array}\right]+\lambda_{3}$ $\left[\begin{array}{ll}\$_{1} & \$_{3}\end{array}\right], \quad\left(\left[\lambda_{1} \$_{1}+\lambda_{2} \$_{2} \$_{3}\right]=\lambda_{1}\left[\begin{array}{ll}\$_{1} & \$_{3}\end{array}\right]+\lambda_{2}\right.$ $\left[\begin{array}{ll}\$_{2} & \$_{3}\end{array}\right]$ ).

Jacobi identity: $\left[\$_{1}\left[\begin{array}{ll}\$_{2} & \$_{3}\end{array}\right]\right]+\left[\$_{3}\left[\begin{array}{ll}\$_{1} & \$_{2}\end{array}\right]\right]+$ $\left[\$_{2}\left[\begin{array}{ll}\$_{3} & \$_{2}\end{array}\right]\right]=(0,0)$.

(4) Killing form: $<\$_{1} ; \$_{2}>=\hat{\mathbf{s}}_{1} \cdot \hat{\mathbf{s}}_{2}$.

(5) Klein form: $\left\{\$_{1} ; \$_{2}\right\}=\hat{\mathbf{s}}_{1} \cdot \mathbf{s}_{\mathrm{O} 2}+\hat{\mathbf{s}}_{2} \cdot \mathbf{s}_{\mathrm{O} 1}$.

Among the operations of the algebra of screws, it is worth to distinguish the Klein form as a fundamental operation concerned with the research at hand. The Klein form is a bilinear symmetric form with useful properties; e.g., it is said that the screws $\$_{1}$ and $\$_{2}$ are reciprocal if $\left\{\$_{1} ; \$_{2}\right\}=0$. A typical case occurs when the primal parts of two screws $\$_{1}$ and $\$_{2}$ associated with revolute joints intersect at a common point. Similarly, assume that $\$_{1}$ and $\$_{2}$ are related with two prismatic joints, then clearly $\left\{\$_{1} ; \$_{2}\right\}=0$. Furthermore, let us consider that $\$_{1}$ represents a revolute joint, while $\$_{2}$ represents a prismatic joint. If the primal part of $\$_{1}$ is perpendicular or parallel to the dual part of $\$_{2}$, then it follows that $\left\{\$_{1} ; \$_{2}\right\}=0$ or $\left\{\$_{1} ; \$_{2}\right\}=1$, respectively.

The solution of the instantaneous kinematics of rigid body in screw form is one of the major contributions of the theory of screws to robot kinematics.

Let us consider a serial kinematic chain with a terminal link $m$ and a base link $j$. Furthermore, let us consider that ${ }^{j} \boldsymbol{\omega}^{m}$ is the angular velocity vector of $m$ as measured from body $j$, while $\mathbf{v}_{O}$ is the linear velocity vector of an arbitrary point $O$ of $m$. The velocity state of body $m$ as measured from the base link $j$ also named a twist about a screw, which is notated as $\mathbf{V}_{O}$, is a six-dimensional vector formed with two concatenated vectors as $\mathbf{V}_{O}=\left(p\left(\mathbf{V}_{O}\right), d\left(\mathbf{V}_{O}\right)\right)=\left({ }^{j} \boldsymbol{\omega}^{m}, v_{O}\right)$ that may be written in screw form as follows:

$$
\begin{aligned}
& { }_{j} \omega_{j+1}{ }^{j} \$^{j+1}+{ }_{j+1} \omega_{j+2}{ }^{j+1} \oiint^{j+2}+\cdots+{ }_{m-2} \omega_{m-1}{ }^{m-2} \oiint^{m-1} \\
& +{ }_{m-1} \omega_{m}{ }^{m-1} \oiint^{m}=\mathbf{V}_{O},
\end{aligned}
$$

where $_{k} \omega_{k+1}(k=j, \ldots, m-1)$ denotes the joint velocity rate between two adjacent bodies $k-1$ and $k$ of the serial chain.

On the contrary, the reduced acceleration state of body $m$ as measured from the base link $j$ is a six-dimensional vector given by $\mathbf{A}_{O}=\left(p\left(\mathbf{A}_{O}\right), d\left(\mathbf{A}_{O}\right)\right)=\left({ }^{j} \boldsymbol{a}^{m}, \mathbf{a}_{O}-{ }^{j} \boldsymbol{\omega}^{m} \times\right.$ $\mathbf{v}_{O}$ ) that may be written in screw form as follows: 


$$
\begin{aligned}
& { }_{j} \alpha_{j+1}{ }^{j} \$^{j+1}+{ }_{j+1} \alpha_{j+2}{ }^{j+1} \$^{j+2}+\cdots+{ }_{m-2} \alpha_{m-1}{ }^{m-2} \$^{m-1} \\
& +{ }_{m-1} \alpha_{m}{ }^{m-1} \oiint^{m}+\mathbf{L}=\mathbf{A}_{O},
\end{aligned}
$$

where ${ }_{k} \alpha_{k+1}=(d / d t)_{k} \omega_{k+1}(k=j, \ldots, m-1)$ denotes the joint acceleration rate between two adjacent bodies $k-1$ and $k$ of the serial chain, ${ }^{j} \alpha^{m}$ is the angular acceleration vector of $m$ as measured from body $j$, and $a_{O}$ is the linear acceleration vector of point $O$. Furthermore,

$$
\begin{aligned}
\mathbf{L}= & {\left[{ }_{j} \omega_{j+1}{ }^{j} \$^{j+1}{ }_{j+1} \omega_{j+2}{ }^{j+1} \$^{j+2}+\cdots+{ }_{m-2} \omega_{m-1}{ }^{m-2} \$^{m-1}+{ }_{m-1} \omega_{m}{ }^{m-1} \$^{m}\right] } \\
& +\cdots+\left[{ }_{m-2} \omega_{m-1}{ }^{m-2} \$^{m-1}{ }_{m-1} \omega_{m}{ }^{m-1} \oiint^{m}\right]
\end{aligned}
$$

is the Lie screw of acceleration of the serial kinematic chain.

Finally, it should be pointed out that both the velocity state and the reduced acceleration state of rigid body in the format here employed meets the rules of helicoidal vector fields [20]. In fact, assuming that $Q$ is another point of $m$ then the velocity and reduced acceleration states taking point $Q$ as the reference pole may be obtained following the condition of the helicoidal vector field as follows:

$$
\begin{aligned}
& \mathbf{V}_{\mathrm{Q}}=\left(p\left(\mathbf{V}_{\mathrm{O}}\right), d\left(\mathbf{V}_{\mathrm{O}}\right)+p\left(\mathbf{V}_{\mathrm{O}}\right) \times \mathbf{r}_{\mathrm{Q} / \mathrm{O}}\right), \\
& \mathbf{A}_{\mathrm{Q}}=\left(p\left(\mathbf{A}_{O}\right), d\left(\mathbf{A}_{O}\right)+p\left(\mathbf{A}_{O}\right) \times \mathbf{r}_{\mathrm{Q} / O}\right),
\end{aligned}
$$

where $\mathbf{r}_{\mathrm{Q} / O}$ is the position vector of $Q$ with respect to $O$.

2.2. The Newton Homotopy Method. The Newton homotopy method [21, 22] is an efficient and elegant method to numerically find the solutions of a square system of polynomials. The problem is formulated as follows: given a system of $n$ polynomials $f(w):=\left\{f_{1}(w), \ldots, f_{n}(w)\right\}: \mathbb{C}^{n} \longrightarrow \mathbb{C}^{n}$, where $w=\left(w_{1}, \ldots, w_{n}\right)$, compute the solutions starting from guessed values of the $n$ variables. For the sake of completeness in what follows, the method is briefly explained.

As an initial step, the original equations must be modified adding new terms in such a way that a new system of equations is formulated. Then, the coefficients of the new system are deformed/perturbed into coefficients of the original system by a series of small increments. To this end, an auxiliary homotopy function $g(w):=\left[g_{1}(w), \ldots\right.$, $\left.g_{n}(w)\right]$ is introduced. Thereafter, the multiple homotopy continuation function $H(w, \bar{t}):=\left[h_{1}(w), \ldots, h_{n}(w)\right]$ is generated as follows:

$$
H(w, \bar{t}):=\left[\begin{array}{c}
h_{1}(w) \\
\vdots \\
h_{n}(w)
\end{array}\right]=\bar{t}\left[\begin{array}{c}
f_{1}(w) \\
\vdots \\
f_{n}(w)
\end{array}\right]+(1-\bar{t})\left[\begin{array}{c}
g_{1}(w) \\
\vdots \\
g_{n}(w)
\end{array}\right],
$$

where $\bar{t} \in[0,1]$ is an arbitrary parameter. In this regard, it is possible to note two boundary conditions:

$$
\begin{aligned}
& H(w, 0)=g(w), \\
& H(w, 1)=f(w) .
\end{aligned}
$$

These conditions ensure convergence of the algorithm owing to the fact that they lead to controllable start systems. Furthermore, an interesting advantage of the method is that there are no restrictions in the selection of the auxiliary homotopy function $g(w)$; e.g., a natural option would be

$$
g(w)=\left[\begin{array}{c}
g_{1}(w) \\
\vdots \\
g_{n}(w)
\end{array}\right]=\left[\begin{array}{c}
\lambda_{1}^{1} w_{1}+\cdots+\lambda_{n}^{1} w_{n}+\lambda^{1} \\
\vdots \\
\lambda_{1}^{n} w_{1}+\cdots+\lambda_{n}^{n} w_{n}+\lambda^{n}
\end{array}\right], \quad \forall \lambda \in \mathbb{R} .
$$

With this in mind, the method consists of solving $H(w, \bar{t})=0$ instead of $f(w)=0$. To this end, in the contribution, the Newton-Raphson technique is applied by varying parameter $\bar{t}$ from 0 to 1 considering small increments of $\bar{t}$. In that concern, the iterations are performed according to

$$
w_{k+1}=w_{k}-\left(J\left(w_{k}\right)\right)^{-1} H\left(w_{k}, \bar{t}\right),
$$

where

$$
J\left(w_{k}\right)=\left[\begin{array}{cccc}
\frac{\partial h_{1}(w)}{\partial w_{1}} & \frac{\partial h_{1}(w)}{\partial w_{2}} & \ldots & \frac{\partial h_{1}(w)}{\partial w_{n}} \\
\vdots & \vdots & \ddots & \vdots \\
\frac{\partial h_{n}(w)}{\partial w_{1}} & \frac{\partial h_{n}(w)}{\partial w_{2}} & \ldots & \frac{\partial h_{n}(w)}{\partial w_{n}}
\end{array}\right]
$$

is the Jacobian matrix of the modified system. The method is easy to follow and the algorithm guarantee convergence due to the fact that undesirable divergence can be avoided.

\section{Description of the Planar Parallel Manipulator with Configurable Platform}

With reference to Figure 1, the PRCP mechanism under study comprises six articulated terminal links $m_{i}$ forming an internal closed-loop chain, named the configurable platform, that is connected to the fixed platform (0) by means of six two-legged limbs. Unless otherwise stated, in the rest of the contribution $i=1,2, \ldots, 6$.

The mobility $M$ of the robot may be determined according to the general formula:

$$
M=d(l-1)-\sum_{i=1}^{j}\left(d-f_{i}\right),
$$

where $d$ is the dimension of the space, $l$ is the number of links (including the fixed link), $j$ is the number of joints, and $f_{i}$ is the dof of the $i$ th joint. For the robot manipulator at hand, we have $d=3, l=19, j=24$, and $f_{i}=1(i=1,2, \ldots, 24)$ that yields $M=6$. This implies that the robot manipulator spans an additional three-dimensional space. In other words, the degree of redundancy of the robot is 3 . Hence, one can install three end-effectors on the configurable platform, a significant advantage over conventional parallel 


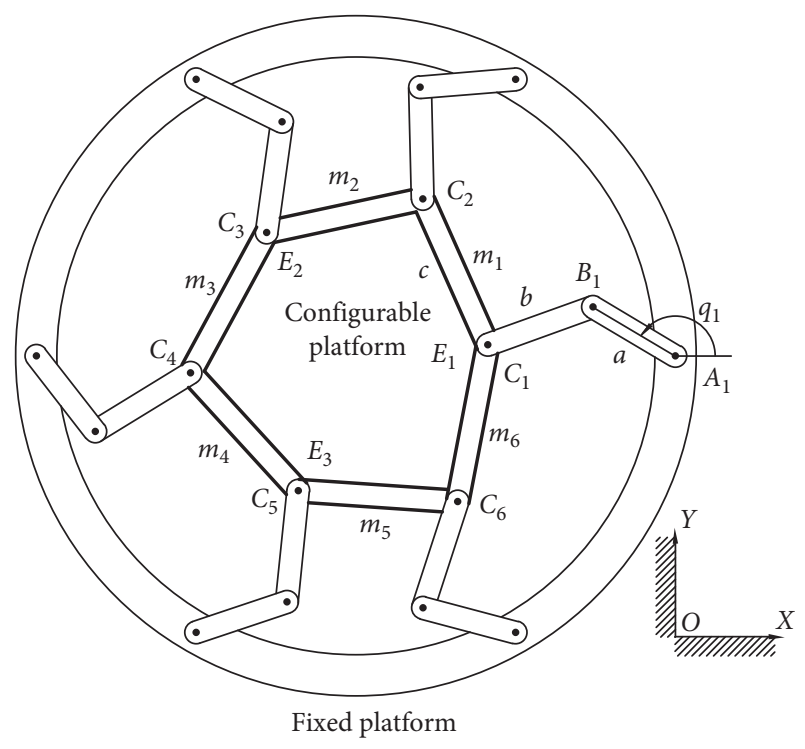

Figure 1: The parallel robot with a configurable platform. An internal closed-loop chain playing the role of a configurable platform is connected to the fixed platform by means of six legs generating a six-degrees-of-freedom configurable robot. Three endeffectors $E_{i}(i=1,2,3)$ are assembled to the internal closed-loop chain taking advantage of three internal or relative degrees of freedom credited to the configurable platform.

manipulators equipped with rigid moving platforms. There are several possibilities to install the end-effectors on the configurable platform, and the option shown in Figure 1 was not considered in previous works [18]. In the following paragraph, the geometry of the PRCP mechanism is explained.

Let us consider that $a$ and $b$ denotes the length of the links forming the $i$ th leg. Furthermore, let $c$ be the length of the terminal links. On the contrary, let us consider that $O_{-} X Y Z$ is a Cartesian coordinate system attached to the fixed platform with unit vectors $\mathbf{i}, \mathbf{j}$, and $\mathbf{k}$. The $i$ th leg is articulated with the fixed platform by means of a revolute joint whose nominal position is denoted by the $i$ th point $A_{i}$ located by vector $\mathbf{a}_{i}$. Similarly, the same leg is articulated with the configurable platform through revolute joints whose nominal positions are specified by the $i$ th point $C_{i}$ located by vector $\mathbf{c}_{i}$. The mobility of the robot demands of the selection of six rotary actuators. Hence, $q_{i}$ denotes the $i$ th generalized coordinate employed to control the orientation of the $i$ th link connecting the $i$ th leg to the fixed platform. Finally, the locations of the endeffectors are denoted by points $E_{i}(i=1,2,3)$ which are located by their corresponding position vectors $\mathbf{e}_{i}(i=1,2,3)$. It is important to emphasize that conveniently the rotary actuators are mounted on the fixed platform. In this regard, advantages over robot manipulators equipped with conventional grippers are evident; e.g., for the PRCP mechanism at hand, the gripping task is achieved by controlling the six coordinates of points $E_{i}(i=1,2,3)$ through handling the six angles $q_{i}$ avoiding the conventional installation of motors over the rigid moving platform.

\section{Displacement Analysis of the PRCP Mechanism}

4.1. Closure Equations. Let us consider that points $C_{i}$ are defined by unknown coordinates $w=\left(w_{1}, \ldots, w_{12}\right)$; i.e., let us consider that $C_{1}=\left(w_{1}, w_{2}\right), \ldots, C_{6}=\left(w_{11}, w_{12}\right)$. Thereafter, the closure kinematic constraints may be written by resorting to the classical Euclidean inner product definition; e.g., upon the parameters $a$ and $b$, evident closure equations may be written as follows:

$$
\begin{aligned}
& \left(\mathbf{b}_{i}-\mathbf{a}_{i}\right) \cdot\left(\mathbf{b}_{i}-\mathbf{a}_{i}\right)=a^{2} \\
& \left(\mathbf{c}_{i}-\mathbf{b}_{i}\right) \cdot\left(\mathbf{c}_{i}-\mathbf{b}_{i}\right)=b^{2}
\end{aligned}
$$

where

$$
\mathbf{b}_{i}=\mathbf{a}_{i}+a \cos \left(q_{i}\right) \hat{\mathbf{i}}+a \sin \left(q_{i}\right) \hat{\mathbf{j}}
$$

Meanwhile, based on the parameter $c$ denoting the length of the terminal links, it follows that

$$
\left(\mathbf{c}_{i}-\mathbf{c}_{i+1}\right) \cdot\left(\mathbf{c}_{i}-\mathbf{c}_{i+1}\right)=c^{2}, \quad i=1, \ldots, 6, \mathbf{c}_{7}=\mathbf{c}_{1} .
$$

On the contrary, the three end-effectors are assembled to the terminal links in such a way that

$$
\begin{aligned}
& \mathbf{e}_{1}=\mathbf{c}_{1}, \\
& \mathbf{e}_{2}=\mathbf{c}_{3}, \\
& \mathbf{e}_{3}=\mathbf{c}_{5} .
\end{aligned}
$$

4.2. Forward Position Analysis. The forward position analysis (FPA) of the PRCP mechanism consists of finding the coordinates of points $E_{i}(i=1,2,3)$ for a prescribed set of generalized coordinates $q_{i}$. In this concern, the computation of the coordinates of points $C_{i}$ is a mandatory task although it would be a boring endeavor. The steps to approach the FPA are as follows:

(1) Based on expressions (13) and (15), generate twelve quadratic equations in the unknowns $w=\left(w_{1}\right.$, $\left.\ldots, w_{12}\right)$

(2) Apply the Newton homotopy method [21, 22] to numerically determine the unknowns $w=\left(w_{1}\right.$, $\left.\ldots, w_{12}\right)$

(3) Taking into account that $C_{1}=\left(w_{1}, w_{2}\right), \ldots$, $C_{6}=\left(w_{11}, w_{12}\right)$, the coordinates of the points $E_{i}(i=$ $1,2,3)$ of the end-effectors are obtained according to expression (16)

The algorithm is easy to follow and guarantees convergence, if any, in a few iterations.

4.3. Inverse Position Analysis. The inverse position analysis (IPA) consists of finding the generalized coordinates $q_{i}$ given the position of the three end-effectors, i.e., given the coordinates of points $E_{i}(i=1,2,3)$. The steps to approach the IPA are as follows: 
(1) Given the position vectors $\mathbf{e}_{i}(i=1,2,3)$, compute the vectors $\mathbf{c}_{i}(i=2,4,6)$ based on equations (15) provided that $\mathbf{c}_{1}=\mathbf{e}_{1}, \mathbf{c}_{3}=\mathbf{e}_{2}$, and $\mathbf{c}_{5}=\mathbf{e}_{3}$. Two quadratic equations are generated for each point $\mathbf{C}_{i}(i=2,4,6)$, and therefore, a closed-form solution is available for the IPA. Thus, the points $\mathbf{C}_{i}(i=$ $2,4,6)$ can reach two positions given the position vectors $\mathbf{e}_{i}(i=1,2,3)$.

(2) Based on expressions (12) and (13), compute the vectors $\mathbf{b}_{i}$. It is straightforward to show that, similar to the previous step, each point $B_{i}$ can reach two positions.

(3) Determine the angles $q_{i}$ upon expression (14).

It is noticeable how easy is to solve the displacement analysis of the PRCP mechanism when the strategy outlined in this section is employed; e.g., existing methods approaching the displacement analysis of similar mechanisms suggest the introduction of orientation angles leading to long trigonometric expressions that are not easy to reduce by means of algebraic elimination methods [18].

\section{Instantaneous Kinematics of the PRCP Mechanism}

In this section, the instantaneous kinematics of the PRCP mechanism is approached by means of the theory of screws [19]. To this end, the infinitesimal screws representing the revolute joints of the PRCP mechanism are depicted in Figure 2.

5.1. Velocity Analysis. The velocity state of the $i$ th terminal link $m_{i}$ as observed from the base 0 taking point $C_{i}$ as the reference pole, e.g., the vector ${ }^{0} \mathbf{V}_{C_{i}}^{m_{i}}=\left({ }^{0} \boldsymbol{\omega}^{m_{i}},{ }^{0} \mathbf{V}_{C_{i}}^{m_{i}}\right)$, may be expressed in screw form through the $i$ th connector chain as follows:

$$
{ }^{0} \mathbf{V}_{C_{i}}^{m_{i}}=\dot{q}_{i}{ }^{0} \$_{i}^{1}+{ }_{1} \omega_{2}^{i 1} \$_{i}^{2}+{ }_{2} \omega_{m_{i}}^{i} \$_{i}^{m_{i}},
$$

where ${ }^{0} \boldsymbol{\omega}^{m_{i}}$ is the angular velocity vector of the $i$ th terminal link $m_{i}$ of the configurable platform as measured from the base $0,{ }^{0} \mathbf{V}_{C_{i}}^{m_{i}}$ is the velocity vector of point $C_{i}$, and ${ }_{0} \omega_{1}^{i}=\dot{q}_{i}$ represents the $i$ th generalized speed. Furthermore, let us consider that $\$_{i}$ is a line in Plücker coordinates directed from $B_{i}$ to $C_{i}$. Then, the application of the Klein form of the $i$ th screw $\$_{i}$ to both sides of expression (17) taking into account that $\left\{\$_{i} ;{ }^{1} \$_{i}^{2}\right\}=\left\{\$_{i} ;{ }^{2} \$_{i}^{m_{i}}\right\}=0$ leads to

$$
\left\{\$_{i} ;{ }^{0} \mathbf{V}_{C_{i}}^{m_{i}}\right\}=\dot{q}_{i}\left\{\$_{i} ;{ }^{0} \$_{i}^{1}\right\}=0
$$

On the contrary, according to the rules of helicoidal vector fields [20], it is possible to establish the following relationship between points and $C_{i+1}$ of the $i$ th terminal link as follows:

$$
\mathbf{V}_{C_{i+1}}=\left(p\left(\mathbf{V}_{C_{i}}\right), d\left(\mathbf{V}_{C_{i}}\right)+p\left(\mathbf{V}_{C_{i}}\right) \times \mathbf{r}_{C_{i+1} / C_{i}}\right),
$$

where $\mathbf{r}_{C_{i+1} / C_{i}}$ is the position vector of point $C_{i+1}$ with respect to $C_{i}$.

Expressions (18) and (19) are sufficient to approach the inverse/forward velocity analysis of the PRCP mechanism, but their organization into a single expression would result

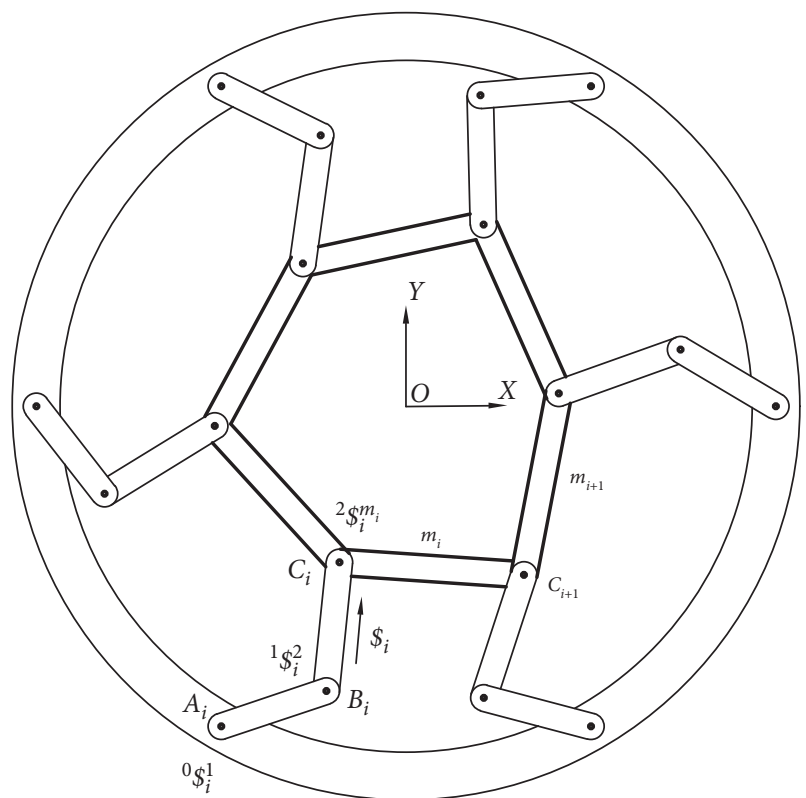

Figure 2: The screws of one leg of the parallel robot with a configurable platform. The screw coordinates of the revolute joints are computed according to the $O_{-} X Y Z$ reference frame taking the origin $O$ of it as the reference pole. Due to the planar kinematics of the problem at hand, evidently three of the six components of each screw vanish. A condition that must be taken properly in order to obtain the input-output equations of velocity and acceleration of the robot.

in an involved procedure. In this regard, consider for instance that for the Gough-Stewart platform, the input/ output equation of velocity may be expressed in compact form as follows [23]:

$$
\underline{J}^{\mathrm{T}} \Delta \mathbf{V}=\left[\begin{array}{c}
\dot{q}_{1} \\
\dot{q}_{2} \\
\vdots \\
\dot{q}_{6}
\end{array}\right],
$$

where $J$ is the $6 \times 6$ Jacobian matrix formed with the lines in Plücker coordinates that are reciprocal to the screws associated with the revolute joints of the robot, while $\Delta$ is the elliptical polar operator that interchanges the primal and dual parts of a screw. Expression (20) is clear, elegant, and easy to implement in computer algorithms, which does not occur for the robot at hand. In fact, casting into a matrixvector form equations (18) and (19) after adjusting the dimension of the velocity states of the terminal links, the input-output equation of velocity of the PRCP mechanism may be expressed as follows:

$$
I_{18 \times 18}\left[\begin{array}{c}
{ }^{0} \mathbf{V}_{C_{1}}^{m_{1}} \\
{ }^{0} \mathbf{V}_{C_{2}}^{m_{2}} \\
\vdots \\
{ }^{0} \mathbf{V}_{C_{6}}^{m_{6}}
\end{array}\right]=J_{18 \times 18}\left[\begin{array}{c}
\dot{q}_{1} \\
\dot{q}_{2} \\
\vdots \\
\dot{q}_{6} \\
0_{12 \times 1}
\end{array}\right] \text {, }
$$

where 


$$
J_{18 \times 18}=\operatorname{diag}\left[\left\{\$_{1} ;{ }^{0} \$_{1}^{1}\right\} \quad\left\{\$_{2} ;{ }^{0} \$_{2}^{1}\right\} \ldots\left\{\$_{1} ;{ }^{0} \$_{6}^{1}\right\} \quad 0 \quad 0 \quad \ldots .0\right]
$$

On the contrary, the direct Jacobian matrix $\underline{J}_{18 \times 18}$ of the PRCP mechanism is given by

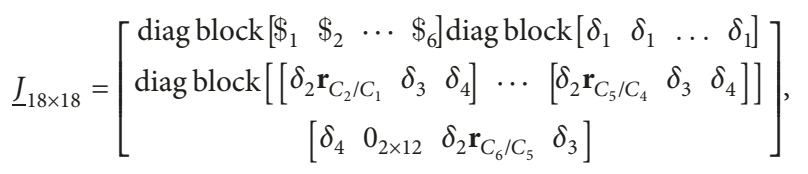

where $\delta_{1}=\left[\begin{array}{lll}0 & 1 & 0 \\ 0 & 0 & 1 \\ 1 & 0 & 0\end{array}\right], \quad \delta_{2}=\left[\begin{array}{cc}0 & 1 \\ -1 & 0\end{array}\right], \quad \delta_{3}=\left[\begin{array}{cc}0 & -1 \\ 0 & -1\end{array}\right]$, and $\delta_{4}=\left[\begin{array}{lll}0 & 1 & 0 \\ 0 & 0 & 1\end{array}\right]$ would be named the planar elliptical polar operators of the configurable robot.

5.2. Acceleration Analysis. The reduced acceleration state of the $i$ th terminal link $m_{i}$ as observed from the base 0 taking point $C_{i}$ as the reference pole, e.g., the vector ${ }^{0} \mathbf{A}_{C_{i}}^{m_{i}}=$ $\left({ }^{0} \boldsymbol{a}^{m_{i}},{ }^{0} \mathbf{a}_{C_{i}}^{m_{i}}-{ }^{0} \boldsymbol{\omega}^{m_{i}} \times{ }^{0} \mathbf{V}_{C_{i}}^{m_{i}}\right)$, may be expressed in screw form through the $i$ th connector chain as follows:

$$
{ }^{0} \mathbf{A}_{C_{i}}^{m_{i}}=\ddot{q}_{i}{ }^{0} \$_{i}^{1}+{ }_{1} \alpha_{2}^{i 1} \$_{i}^{2}+{ }_{2} \alpha_{m_{i}}^{i}{ }^{2} \$_{i}^{m_{i}}+\mathbf{L}_{i}
$$

where ${ }^{0} \boldsymbol{\alpha}^{m_{i}}$ is the angular acceleration vector of the $i$ th terminal link $m_{i}$ of the configurable platform as measured from the base $0,{ }^{0} \mathbf{a}_{C_{i}}^{m_{i}}$ is the acceleration vector of point $C_{i}$, and ${ }_{0} \omega_{1}^{i}=\ddot{q}_{i}$ represents the $i$ th generalized acceleration. Furthermore,

$$
\mathbf{L}_{i}=\left[\dot{q}_{i}{ }^{0} \$_{i}^{1}{ }_{1} \omega_{2}^{i 1} \$_{i}^{2}+{ }_{2} \omega_{m_{i}}^{i}{ }^{2} \$_{i}^{m_{i}}\right]+\left[{ }_{1} \omega_{2}^{i 1} \$_{i}^{2}{ }_{2} \omega_{m_{i}}^{i}{ }^{2} \$_{i}^{m_{i}}\right]
$$

is the $i$ th Lie screw of acceleration.

Finally, following the trend of the velocity analysis, the input-output equation of acceleration of the configurable robot results in

$$
I_{18 \times 18}\left[\begin{array}{c}
{ }^{0} \mathbf{A}_{C_{1}}^{m_{1}} \\
{ }^{0} \mathbf{A}_{C_{2}}^{m_{2}} \\
\vdots \\
{ }^{0} \mathbf{A}_{C_{6}}^{m_{6}}
\end{array}\right]=J_{18 \times 18}\left[\begin{array}{c}
\ddot{q}_{1} \\
\ddot{q}_{2} \\
\vdots \\
\ddot{q}_{6} \\
0_{12 \times 1}
\end{array}\right]+\left[\begin{array}{c}
\left\{\$_{1} ; \mathbf{L}_{1}\right\} \\
\left\{\$_{2} ; \mathbf{L}_{2}\right\} \\
\vdots \\
\left\{\$_{6} ; \mathbf{L}_{6}\right\} \\
0_{12 \times 1}
\end{array}\right] .
$$

It is noticeable how the acceleration analysis of robot manipulators can be easily formulated when the theory of screws is employed.

\section{Changing the Operation Mode of the PRCP Mechanism}

Besides the evident advantages of having a configurable platform, the versatility of the PRCP mechanism under study is such that we can propose alternative operation modes, as shown in Figure 3. Two opposite terminal links of the robot may be selected as two moving platforms able to adopt arbitrary poses as observed from the fixed platform.

The kinematics of the PRCP mechanism introduced in Figure 3 is closer to the outlined for the PRCP mechanism with three-end effectors, and therefore, it is straightforward to include their kinematic analyses in the contribution.

\section{Numerical Application}

This section is devoted to numerically exemplify the method of kinematic analysis developed for the proposed parallel manipulator with configurable platform. To this end, the parameters of the configurable robot are given by $a=180 \mathrm{~mm}, b=240 \mathrm{~mm}$, and $c=160 \mathrm{~mm}$. Furthermore, the points $A_{i}$ are equally spaced on a circumference of radius $r=380 \mathrm{~mm}$. Hence, accordingly the reference frame $O_{-} X Y Z$ attached to the center of the fixed platform, the coordinates of these points are given by $A_{1}=(380,0) \mathrm{mm}$, $A_{2}=(190,329.089) \mathrm{mm}, A_{3}=(-190,329.089) \mathrm{mm}, A_{4}=$ $(-380,0) \mathrm{mm}, A_{5}=(-190,-329.089) \mathrm{mm}$, and $A_{6}=(190$, $-329.089) \mathrm{mm}$. In what follows, most of the topics treated in the contribution are illustrated through numerical applications.

7.1. Case Study 1: Forward Kinematics. The case study 1 is devoted to numerically exemplify the solution of the forward kinematic problem of the terminal links $m_{i}$ of the robot manipulator.

7.1.1. Forward Position Analysis: Numerical Solution. Let us consider that, in addition to the given parameters of the mechanism, the generalized coordinates associated with the actual configuration of the robot are given by $q_{1}=130^{\circ}$, $q_{2}=200^{\circ}, q_{3}=320^{\circ}, q_{4}=320^{\circ}, q_{5}=45^{\circ}$, and $q_{6}=160^{\circ}$. Following the steps indicated in Section 4.2, the application of expressions (13) and (15) yields the following 12 quadratic equations:

$$
\begin{aligned}
& f_{1}=w_{1}^{2}-528.5964604 w_{1}+31266.65495+w_{2}^{2}-275.7759994 w_{2} \\
& f_{2}=w_{3}^{2}-41.7106568 w_{3}+14405.12016+w_{4}^{2}-535.0520552 w_{4} \\
& f_{3}=w_{5}^{2}+104.2240004 w_{5}-9349.95052+w_{6}^{2}-426.7757674 w_{6} \\
& f_{4}=w_{7}^{2}+484.2240006 w_{7}+14405.12027+w_{8}^{2}+231.4035400 w_{8} \\
& f_{5}=w_{9}^{2}+125.4415586 w_{9}-12938.65301+w_{10}^{2}+403.6208658 w_{10} \\
& f_{6}=w_{11}^{2}-41.7106566 w_{11}+14405.12016+w_{12}^{2}+535.0520552 w_{12} \\
& f_{7}=w_{1}^{2}-2 w_{1} w_{3}+w_{3}^{2}+w_{2}^{2}-2 w_{2} w_{4}+w_{4}^{2}-25600.00 \\
& f_{8}=w_{3}^{2}-2 w_{3} w_{5}+w_{5}^{2}+w_{4}^{2}-2 w_{4} w_{6}+w_{6}^{2}-25600.00 \\
& f_{9}=w_{5}^{2}-2 w_{5} w_{7}+w_{7}^{2}+w_{6}^{2}-2 w_{6} w_{8}+w_{8}^{2}-25600.00 \\
& f_{10}=w_{7}^{2}-2 w_{7} w_{9}+w_{9}^{2}+w_{8}^{2}-2 w_{8} w_{10}+w_{10}^{2}-25600.00 \\
& f_{11}=w_{9}^{2}-2 w_{9} w_{11}+w_{11}^{2}+w_{10}^{2}-2 w_{10} w_{12}+w_{12}^{2}-25600.00 \\
& f_{12}=w_{11}^{2}-2 w_{11} w_{1}+w_{1}^{2}+w_{12}^{2}-2 w_{12} w_{2}+w_{2}^{2}-25600.00
\end{aligned}
$$

In order to find one solution of the quadratic equations, the Newton homotopy method was employed. To this aim, 


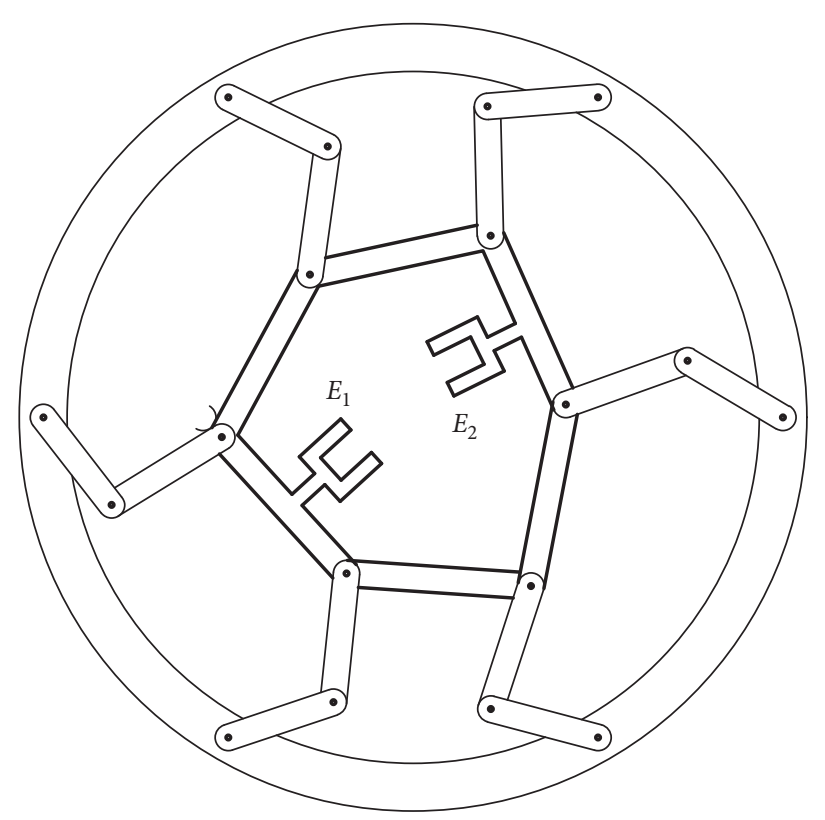

Figure 3: Two opposite terminal links assume the role of two end-effectors, $E_{1}$ and $E_{2}$, able to adopt arbitrary poses. Thereby, the configurable parallel manipulator is designed to work as two cooperative robots. This mechanical device is kinematically equivalent to two conventional three-degrees-of-freedom planar parallel manipulators. However, the rigidity and accuracy would be improved due to the fact that the end-effectors $E_{1}$ and $E_{2}$ are interconnected through the remaining terminal links.

TABLE 1: One solution of the forward displacement problem using the Newton homotopy method.

\begin{tabular}{lccccccccccccc}
\hline Iter & $f_{1}$ & $w_{1}$ & $w_{2}$ & $w_{3}$ & $w_{4}$ & $w_{5}$ & $w_{6}$ & $w_{7}$ & $w_{8}$ & $w_{9}$ & $w_{10}$ & $w_{11}$ & $w_{12}$ \\
\hline 1 & -9322.783 & 100.0 & -8.0 & -50.0 & 40.0 & -200.00 & 60.0 & -50.0 & 60.0 & 60.0 & -10.0 & -100.0 & -20.0 \\
2 & 2211.322 & 56.271 & 9.295 & -97.730 & 53.080 & -256.512 & 74.713 & -96.179 & 81.528 & 52.003 & 10.100 & -103.998 & -58.868 \\
3 & 455.409 & 71.083 & -6.067 & -79.658 & 48.798 & -239.373 & 61.746 & -79.256 & 62.425 & 68.396 & 0.121 & -84.390 & -50.793 \\
4 & 14.890 & 74.076 & -8.503 & -75.710 & 47.772 & -235.378 & 58.336 & -75.371 & 57.045 & 72.704 & -3.587 & -80.502 & -49.942 \\
5 & 0.005 & 74.058 & -8.429 & -75.728 & 47.818 & -235.376 & 58.423 & -75.383 & 56.929 & 72.698 & -3.664 & -80.457 & -49.958 \\
6 & $-0.2 e-6$ & 74.058 & -8.430 & -75.728 & 47.817 & -235.376 & 58.422 & -75.383 & 56.929 & 72.698 & -3.665 & -80.457 & -49.958 \\
\hline
\end{tabular}

In only six iterations, the algorithm was able to converge to one real solution.

the auxiliary functions of homotopy were chosen as it was recommended in equations (8); e.g., one of the twelve functions $g_{i}$ is selected as

$$
\begin{aligned}
g_{1}= & w_{1}+w_{2}+w_{3}+w_{4}+w_{5}+w_{6}+w_{7}+w_{8}+w_{9}+w_{10} \\
& +w_{11}+w_{12} .
\end{aligned}
$$

There is nothing special in this selection. Thereafter, starting from guessed solutions for the unknowns $w_{i}(i=$ $1,2,3, \ldots, 12)$, the Newton homotopy algorithm yields the numerical results listed in Table 1.

It is noticeable that, according to Table 1, in only six iterations, the algorithm was able to reduce the error of function $f_{1}$ from -9322.783 to $-0.2 e-6$. Similar errors were found for the remaining functions $f_{i}$. Furthermore, by changing the initial guessed values, the method was able to find 22 poses of the configurable closed-loop chain. The coordinates of the corresponding points $C_{i}$ are listed in Table 2 .

Finally, it is opportune to cite that positioning errors in a parallel manipulator can restrict achieving the required quality of the part [24]. Hence, the implementation of a trusted strategy to solve the inherent position analysis of parallel manipulators becomes a crucial task.

7.1.2. Forward Velocity and Acceleration Analyses of the Terminal Links. The next part of the case study 1 is dedicated to compute the instantaneous kinematics of the PRCP mechanism; e.g., it is required to compute the velocity and acceleration of points $C_{i}$. To this aim, solution 16 of Table 2 is chosen as the reference configuration of the robot manipulator. Furthermore, let us consider that, upon the reference configuration of the robot, the rotary actuators are commanded to follow periodical functions given by $q_{i}(t)=q_{i}+0.2 \sin (t) \cos (t)$, where the time $t$ is given in the interval $0 \leq t \leq 2 \pi(\mathrm{s})$. With this in mind, the application of the method introduced in Section 5 yields the solution of the instantaneous kinematics of the PRCP mechanism which is summarized in Figure 4.

7.2. Case Study 2: Inverse Kinematics. The case study 2 is devoted to apply the method developed for the inverse position analysis of the robot manipulator. Dealing with the 
TABLE 2: Resulting coordinates of points $C_{i}$.

\begin{tabular}{|c|c|c|c|c|c|c|}
\hline Sol. & $C_{1} \mathrm{~mm}$ & $C_{2} \mathrm{~mm}$ & $C_{3} \mathrm{~mm}$ & $C_{4} \mathrm{~mm}$ & $C_{5} \mathrm{~mm}$ & $C_{6} \mathrm{~mm}$ \\
\hline 1 & $(74.058,-8.430)$ & $(-75.728,47.817)$ & $(-235.376,58.422)$ & $(-75.383,56.929)$ & $(72.698,-3.665)$ & $(-80.457,-49.958)$ \\
\hline 2 & $(31.597,79.151)$ & $(-128.400,79.584)$ & $(-278.730,134.370)$ & $(-124.100,93.278)$ & $(-228.330,-28.109)$ & $(-69.236,-49.958)$ \\
\hline 3 & $(91.481,-28.647)$ & $(-53.377,39.295)$ & $(106.510,33.279)$ & $(-53.490,32.695)$ & $(96.717,-22.424)$ & $(216.490,-128.510)$ \\
\hline 4 & $59.536,2.353)$ & $(-81.500,50.447)$ & $(72.909,8$ & $(-78.593$ & $(69.202,-1.319)$ & $(-82.860,-5$ \\
\hline 5 & $(63.650,6.204)$ & & & $(-3.693,-143.210)$ & & $(-85.281,-52.270)$ \\
\hline 6 & $(108.850,-44.963)$ & $(187.210,94.533)$ & & $(-4.196,-147.260)$ & $(113.930,-39.351)$ & $(229.890,-149.600)$ \\
\hline 7 & $59.250,-77.899)$ & $(39.532,28.254)$ & $(-113.440,-18$ & $(-2.830,-134$ & $(152.360,-95$ & $(7.255,-27.912)$ \\
\hline 8 & $56.314,18.128)$ & $(-98.323,59.2$ & $(-255.940,8$ & $(-96.374,74$ & $(49.893,10$. & $(177.820,-8$ \\
\hline 9 & $(161.110,-78.7$ & & $(-110.2$ & & $(148.82,-88$ & $(253.680,-209.290)$ \\
\hline 10 & $(108.630,-44.783)$ & $(187.250,94.572)$ & & & $(108.680,-33$ & $(-51.247,-38.613)$ \\
\hline 11 & $(98.320,-35.465)$ & $(189.05,96.323)$ & $(60.294,1.3$ & $(--85.853,66$. & $(-220.09,-20.606)$ & $(-61.542,-42.114)$ \\
\hline 12 & $61.143,10.108)$ & $(193.18,100.481)$ & $(65.430,4.142)$ & $(-82.972,63.949)$ & $(64.437,1.735)$ & $(-86.038,-52.645)$ \\
\hline 13 & $(65$ & & & & & 7) \\
\hline 14 & $(113.58,-48.889)$ & $(-24.545,31.859)$ & $(-183.36,12.458)$ & $(-305.54,115.761)$ & $(-203.17,-7.198)$ & $(-45.974,-37.018)$ \\
\hline 15 & $(66.804,1.520)$ & $(-84.983,52.124)$ & $(68.128,5.680)$ & $(-81.420,62.562)$ & & $(191.49,-98.751)$ \\
\hline 16 & $31.302,80.33$ & $(-128.70,79.819)$ & $(-5.2725,-21$. & $(-119.14,90.402)$ & $(-227.50,-27.320)$ & $(-68.456,-44.763)$ \\
\hline 17 & & $(-88$ & & $(-3$ & $(61$ & $(18$ \\
\hline 18 & $(27.358,99.685)$ & $(187.28,94.599)$ & & $(-4.186,-147.191)$ & $(107.43,-32.547)$ & $(-52.444,-38.993)$ \\
\hline 19 & $(53.904,22.413)$ & $(193.42,100.731)$ & $(65.739,4.315)$ & $(-3.3453,-140.001)$ & $(45.740,12.284)$ & $(174.29,-82.978)$ \\
\hline 20 & $(29.191,89.673)$ & & $(60.298,1.340)$ & & $(-220.09,-20.605)$ & $(-61.541,-42.113)$ \\
\hline 21 & $(46.406,37.274)$ & & $(65.617,4.247)$ & & $(64.553,1.662)$ & $(-85.962,-52.607)$ \\
\hline 22 & $(91.086,-28.237)$ & $(-53.887,39.461)$ & $(-213.85,36.073)$ & $(-53.877,33.186)$ & $(96.289,-22.044)$ & $(216.151,-128.03)$ \\
\hline
\end{tabular}

The method employed in the contribution yields 22 real solutions for the forward position analysis.
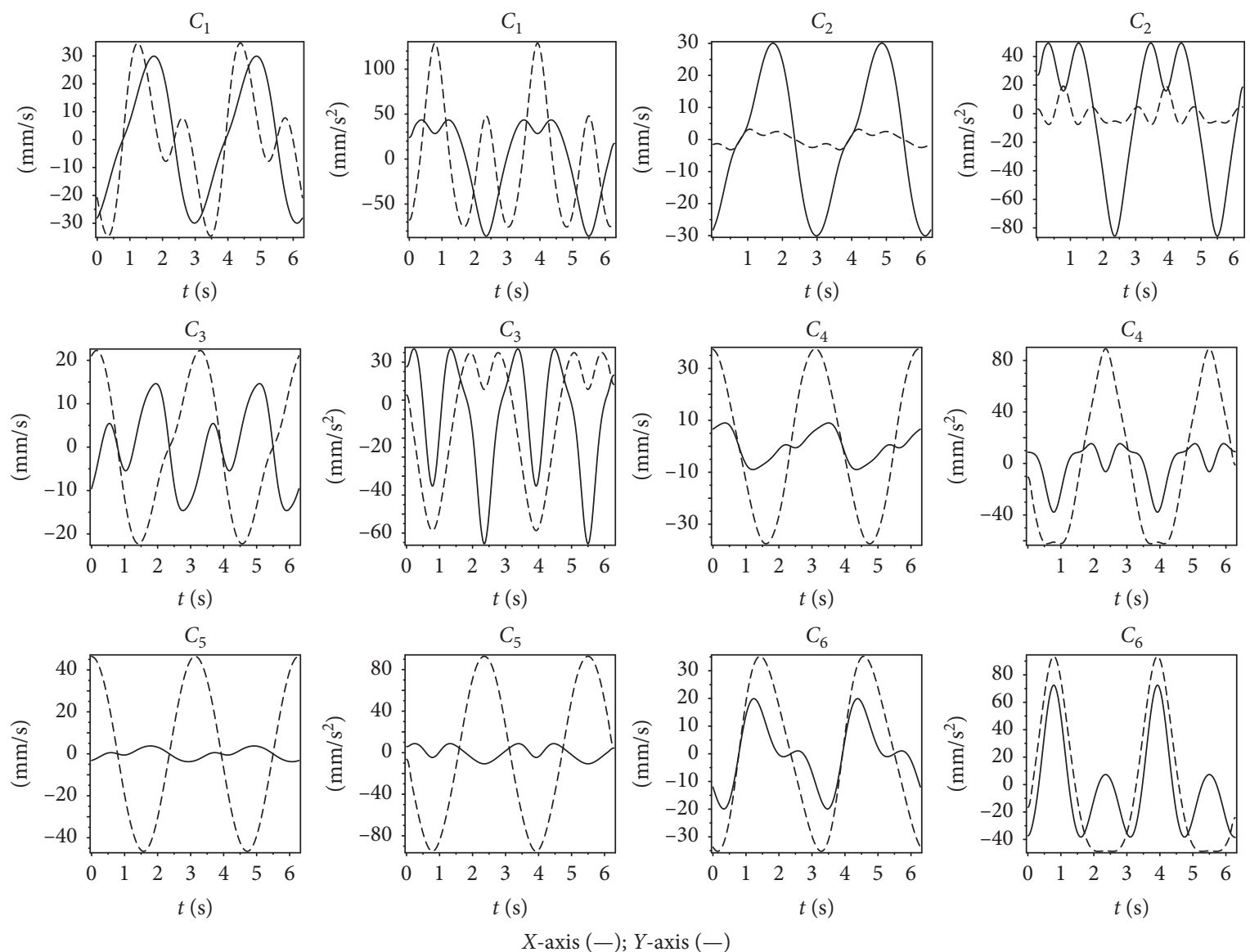

FIGURE 4: Instantaneous kinematics of the PRCP mechanism. Upon the reference configuration of the robot, see solution 16 of Table 2, the rotary actuators must follow periodical functions given by $q_{i}(t)=q_{i}+0.2 \sin (t) \cos (t)$. The linear velocity and acceleration of points $C_{i}$ are presented in different plots according to the $X$ and $Y$ axes, for clarity. 


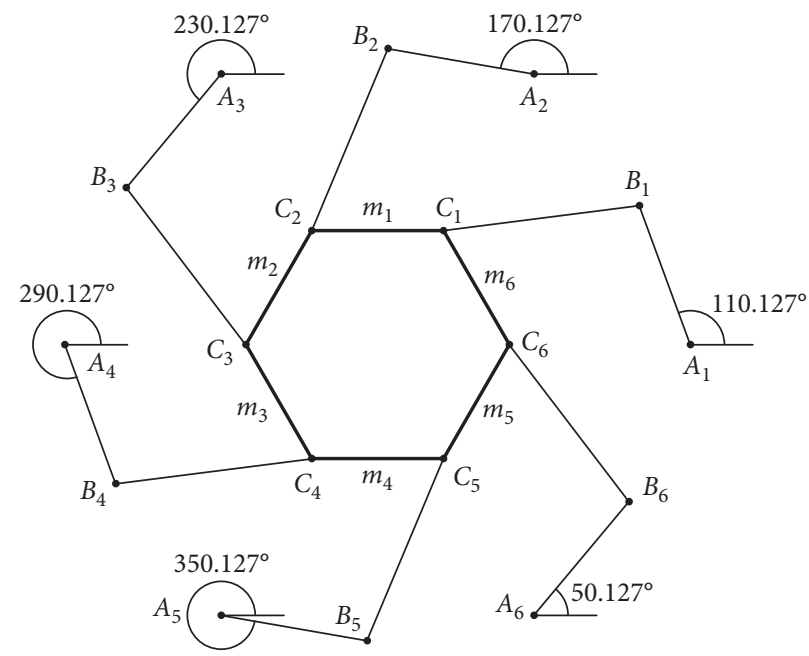

FIGURE 5: Case study 2. Reference configuration of the PRCP mechanism. The reason for this configuration is simple. A symmetric posture is strongly advisable for any parallel manipulator. Furthermore, such postures are referred as optimized reference configurations [5].
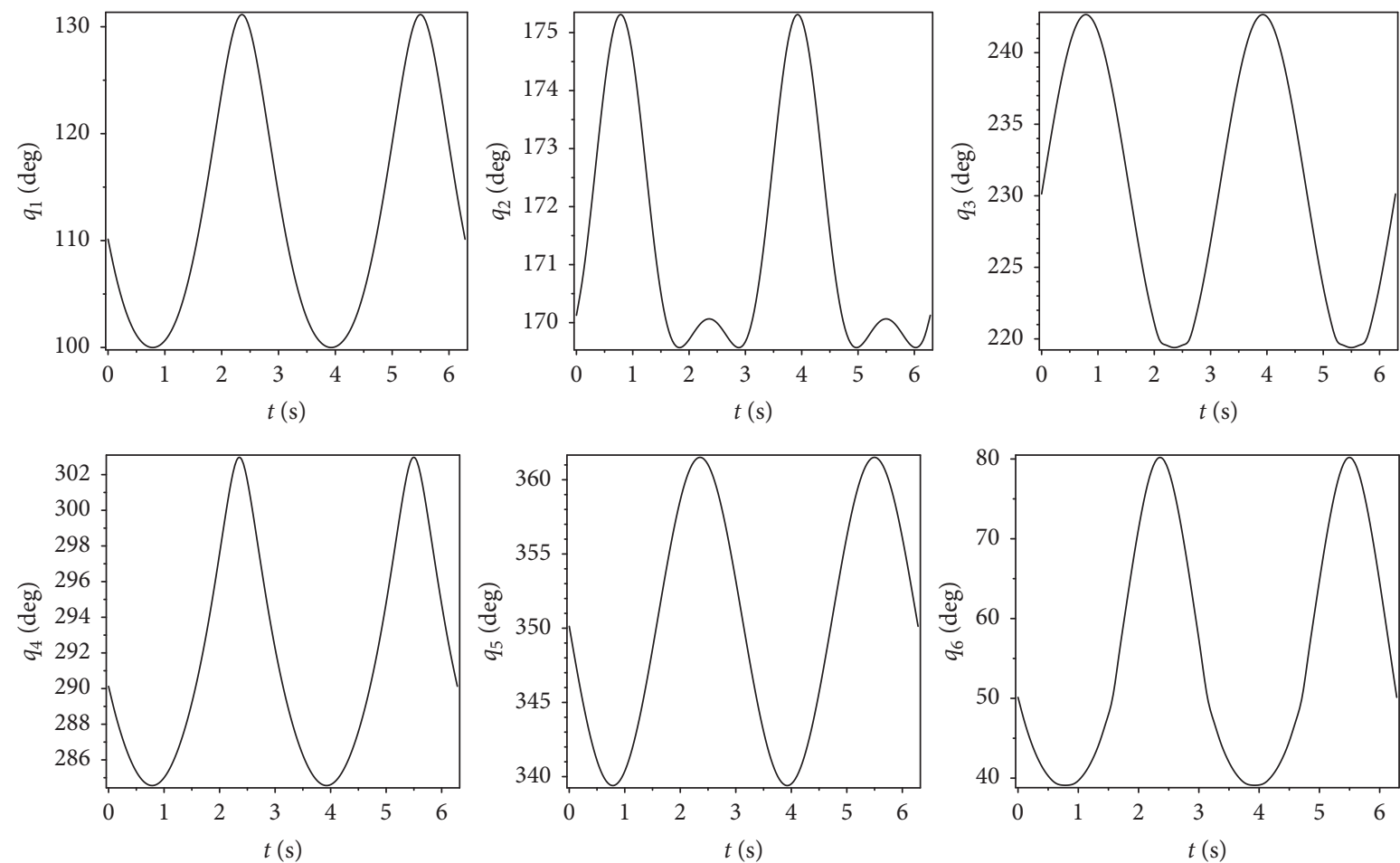

FIGURE 6: Example 2. Inverse position analysis. Upon the reference configuration of the robot, the end-effectors or points $E_{i}(i=1,3,5)$ must follow periodical functions of the type $\sin (t) \cos (t)$. At the time $t=0$, the robot is in the configuration, as shown in Figure 5 , and after $2 \pi(\mathrm{s})$, the robot returns to its original posture.

initial posture of the robot, it is worth to note that, in parallel manipulators with configurable platforms, optimized reference configurations are related with symmetric postures of the robot [5]. Hence, for the case study 2, as shown in Figure 5, a symmetric posture is selected as the reference configuration of the robot.

7.2.1. Robot Manipulator Equipped with Three End-Effectors. Let us consider that, upon the reference configuration of the robot, the three end-effectors are commanded to follow periodical functions given by $E_{1}=(100 \sin t \cos t,-120$ $\sin t \cos t) \mathrm{mm}, E_{2}=(70 \sin t \cos t,-40 \sin t \cos t) \mathrm{mm}$, and $E_{3}=(-30 \sin t \cos t,-60 \sin t \cos t) \mathrm{mm}$, where the time $t$ is given in the interval $0 \leq t \leq 2 \pi$ (s). The resulting time history of the orientation of the generalized coordinates $q_{i}$ meeting such conditions is provided in Figure 6.

7.2.2. Robot Manipulator Equipped with Two End-Effectors. Let us consider that, upon the reference configuration of the robot, see Figure 5, the position and orientation of the 
Table 3: Trajectories imposed to the terminal links $m_{2}$ and $m_{5}$.

\begin{tabular}{lccc}
\hline Terminal link & $\delta_{X}$ & $\delta_{Y}$ & $\delta_{\theta}$ \\
\hline$m_{2}$ & $-3.75\left[\cos ^{3}(2 t-\pi / 2)+2 \sin (t / 2)\right]$ & $7.5 \sin (t) \cos (t)$ & $0.1 \sin (t) \cos (t)$ \\
$m_{5}$ & $3.75 \sin (t) \cos (t)$ & $-8.75\left[\cos ^{3}(2 t+\pi / 2)+2 \sin (t / 2)\right]$ & $0.15\left[\sin ^{2}(2 t) \cos ((t / 2)-(\pi / 2))\right]$ \\
\hline
\end{tabular}

The pose of $m_{2}$ does not depend on the pose of $m_{5}$ enhancing the six-degrees-of-freedom of the configurable robot. The time $t$ is given in the interval $0 \leq t \leq 2 \pi(\mathrm{s})$.
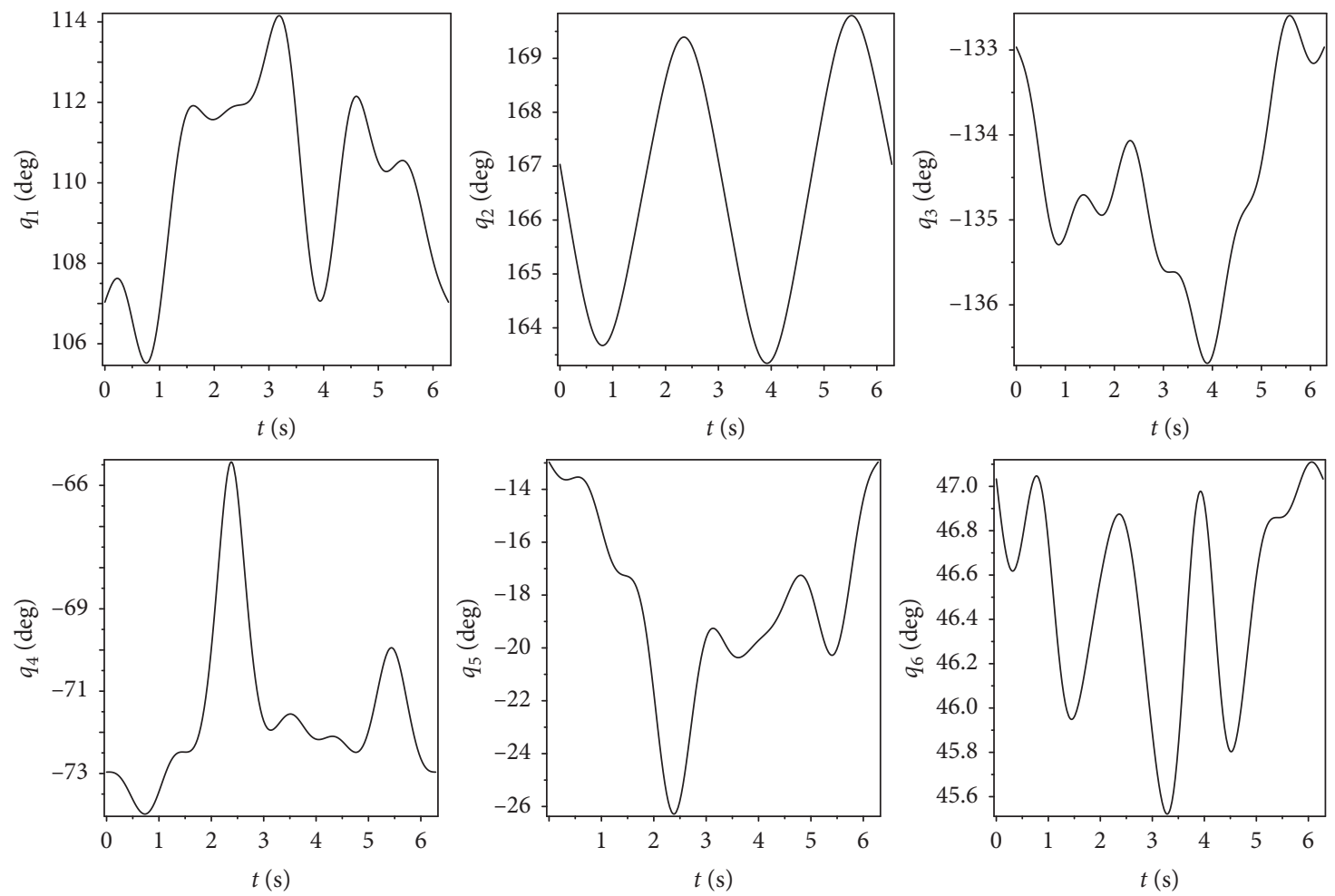

FigURE 7: Temporal behavior of the displacement analysis of the generalized coordinates $q_{i}$. For clarity, the results are reported in different graphics. At the beginning of the motion, the robot manipulator is in the reference configuration, as shown in Figure 6 , and after $2 \pi$ (s), the robot returns to its reference configuration.

end-effectors $m_{2}$ and $m_{5}$ are constrained to instantaneously change according to the increments/decrements given in Table 3.

Thereafter, the resulting temporal behavior of the orientation of the rotary actuators $q_{i}$ meeting such conditions is shown in Figure 7.

\section{Conclusions}

Recently, the concept of configurable platform was introduced in the field of parallel manipulators as an option for the development of gripping robots with high dynamic performance $[1,25]$. Advantages like improved workspace, reduction of singularities, and the performance of simultaneous tasks due to the assembly of multiple end-effectors are without doubt attributes that make the development of this new class of robot manipulators an extremely attractive research field. The opportunities and applications of such robots are very promissory. Motivated by this trend, in this work, a planar parallel manipulator with a configurable platform is introduced.
The proposed robot consists of six legs sharing an internal closed-loop chain, named the configurable platform, formed with six terminal links connected by means of revolute joints. The mobility of the robot is such that three end-effectors are assembled to the robot enhancing its dexterity; e.g., the end-effectors are able to adopt arbitrary positions performing simultaneous tasks. The forward displacement analysis leads us twelve quadratic equations which are solved by means of the Newton homotopy method. Furthermore, the simplicity of the topology of the robot at hand is such that it is possible to obtain a closedform solution for the inverse displacement analysis. On the contrary, the input-output equations of velocity and acceleration of the robot are obtained by resorting to reciprocal-screw theory. The versatility of the robot manipulator is such that we can modify the operation mode; e.g., two three-degrees-of-freedom end-effectors can be employed instead of the original three two-degrees-offreedom end-effectors. Finally, numerical examples are provided with the purpose to exemplify the method of kinematic analysis. 
Finally, for future work, elucidating topics like optimization, manipulability indexes, and grasping concerned with the parallel manipulator with a configurable platform proposed in the contribution would enhance the attributes of this robot.

\section{Data Availability}

The Maple sheet data used to support the findings of this study are available from the author upon request.

\section{Conflicts of Interest}

The authors declare that they have no conflicts of interest.

\section{Acknowledgments}

The first author gratefully acknowledges with thanks the support of the National Council of Science and Technology of Mexico (CONACYT) through the National Network of Researchers fellowship (Grant number 7903).

\section{Supplementary Materials}

The supplementary materials are two animations (videos in avi format) of the robot which was a recommendation of one of the reviewers. (Supplementary Materials)

\section{References}

[1] P. Lambert and J. L. Herder, "Parallel robots with configurable platforms: fundamental aspects of a new class of robotic architectures," Proceedings of the Institution of Mechanical Engineers, Part C: Journal of Mechanical Engineering Science, vol. 230, no. 1, pp. 11-47, 2016.

[2] A. K. Dash, I.-M. Chen, S. H. Yeo, and G. Yang, "Taskoriented configuration design for reconfigurable parallel manipulator systems," International Journal of Computer Integrated Manufacturing, vol. 18, no. 7, pp. 615-634, 2005.

[3] Z. Li and R. Katz, "A reconfigurable parallel kinematic drilling machine and its motion planning," International Journal of Computer Integrated Manufacturing, vol. 18, no. 7, pp. 610614, 2005.

[4] W. Haouas, R. Dahmouche, N. Le Fort-Piat, and G. J. Laurent, "A new seven degrees-of-freedom parallel robot with a foldable platform," ASME Journal of Mechanisms and Robotics, vol. 10, no. 4, 2018.

[5] B.-J. Yi, H. Y. Na, J. H. Lee et al., "Design of a parallel-type gripper mechanism," The International Journal of Robotics Research, vol. 21, no. 7, pp. 661-676, 2002.

[6] M. G. Mohamed and C. M. Gosselin, "Design and analysis of kinematically redundant parallel manipulators with configurable platforms," IEEE Transactions on Robotics, vol. 21, no. 3, pp. 277-287, 2005.

[7] A. G. Hoevenaars, P. Lambert, and J. L. Herder, "Kinematic design of two elementary 3DOF parallel manipulators with configurable platforms," in Computational Kinematics. Mechanisms and Machine Science, F. Thomas and A. PérezGracia, Eds., pp. 315-322, Springer, Dordrecht, Netherlands, 2014.
[8] K.-C. Lee, H.-P. Huang, and S.-S. Lu, "A flexible robot inspection cell based on vision image," International Journal of Computer Integrated Manufacturing, vol. 5, no. 4-5, pp. 310-318, 1992.

[9] P. Gil, J. Pomares, S. V. T. Puente, C. Diaz, F. Candelas, and F. Torres, "Flexible multi-sensorial system for automatic disassembly using cooperative robots," International Journal of Computer Integrated Manufacturing, vol. 20, no. 8, pp. 757-772, 2007.

[10] N. Papakostas, G. Michalos, S. Makris, D. Zouzias, and G. Chryssolouris, "Industrial applications with cooperating robots for the flexible assembly," International Journal of Computer Integrated Manufacturing, vol. 24, no. 7, pp. 650660, 2011.

[11] G. Coppola, D. Zhang, and K. Liu, "A 6-DOF reconfigurable hybrid parallel manipulator," Robotics and Computer-Integrated Manufacturing, vol. 30, no. 2, pp. 99-106, 2014.

[12] V. Venkataramanujam and P. M. Larochelle, "Design and development of planar reconfigurable motion generators," Mechanics Based Design of Structures and Machines, vol. 44, no. 4, pp. 426-439, 2016.

[13] S. Herrero, T. Mannheim, I. Prause, C. Pinto, B. Corves, and O. Altuzarra, "Enhancing the useful workspace of a reconfigurable parallel manipulator by grasp point optimization," Robotics and Computer-Integrated Manufacturing, vol. 31, no. 2, pp. 51-60, 2015.

[14] J. A. Kereluk and M. R. Emami, "Task-based optimization of reconfigurable robot manipulators," Advanced Robotics, vol. 31, no. 16, pp. 836-850, 2017.

[15] T. Panagiota, M. Alexandros-Stereos, M. Sotiris, and G. Chryssolouris, "On a human-robot collaboration in an assembly cell," International Journal of Computer Integrated Manufacturing, vol. 30, no. 6, pp. 580-589, 2017.

[16] G. Huang, S. Guo, D. Zhang, H. Qu, and H. Tang, "Kinematic analysis and multi-objective optimization of a new reconfigurable parallel mechanism with high stiffness," Robotica, vol. 36, no. 2, pp. 187-203, 2018.

[17] W. Saab, P. Racioppo, and P. Ben-Tzvi, "A review of coupling mechanism designs for modular reconfigurable robots," Robotica, vol. 37, no. 2, pp. 378-403, 2019.

[18] G. Li, D. Song, S. Xu, L. Sun, and J. Liu, "A hybrid model and model-free position control for a reconfigurable manipulator," IEEE/ASME Transactions on Mechatronics, vol. 24, no. 2, pp. 785-795, 2019.

[19] J. Gallardo-Alvarado, Kinematic Analysis of Parallel Manipulators by Algebraic Screw Theory, Springer International Publishing, New York, NY, USA, 2016.

[20] J. Gallardo-Alvarado and J. M. Rico-Martinez, "Screw theory and helicoidal fields," in Proceedings of the ASME 1998 Design Engineering Technical Conferences (DETC98/MECH-5893), Atlanta, Georgia, September 1998.

[21] T.-M. Wu, "A study of convergence on the Newton-homotopy continuation method," Applied Mathematics and Computation, vol. 168, no. 2, pp. 1169-1174, 2005.

[22] J. Gallardo-Alvarado, "An application of the Newtonhomotopy continuation method for solving the forward kinematic problem of the 3-RRS parallel manipulator," Mathematical Problems in Engineering, vol. 2019, Article ID 3123808, 6 pages, 2019.

[23] J. Gallardo, J. M. Rico, A. Frisoli, D. Checcacci, and M. Bergamasco, "Dynamics of parallel manipulators by means of screw theory," Mechanism and Machine Theory, vol. 38, no. 11, pp. 1113-1131, 2003. 
[24] M. Sohail, S. U. Butt, and A. A. Baqai, "An analytical approach for positioning error and mode shape analysis of $n$-legged parallel manipulator," International Journal of Computer Integrated Manufacturing, vol. 31, no. 8, pp. 677-691, 2018.

[25] A. G. L. Hoevenaars, C. Gosselin, P. Lambert, and J. L. Herder, "A systematic approach for the Jacobian analysis of parallel manipulators with two end-effectors," Mechanism and Machine Theory, vol. 109, no. 1, pp. 171-194, 2017. 


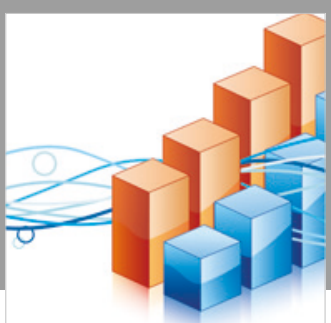

Advances in

Operations Research

\section{-n-m}
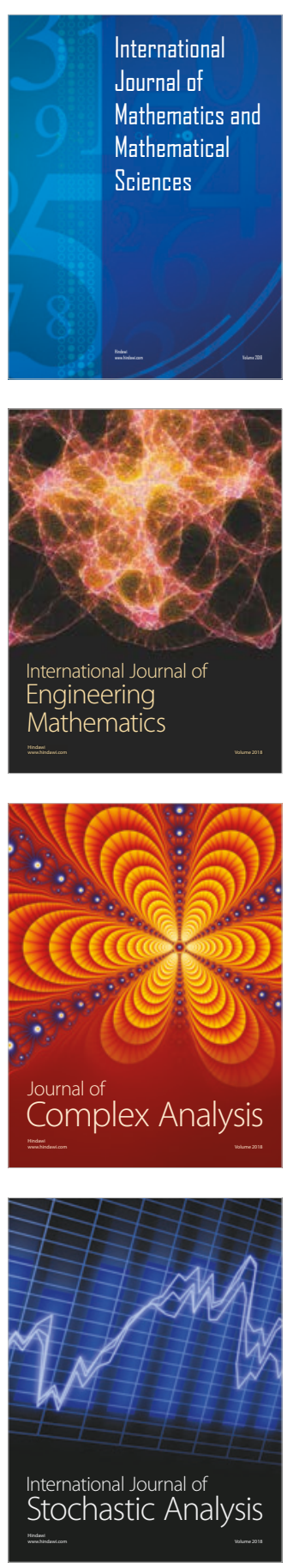
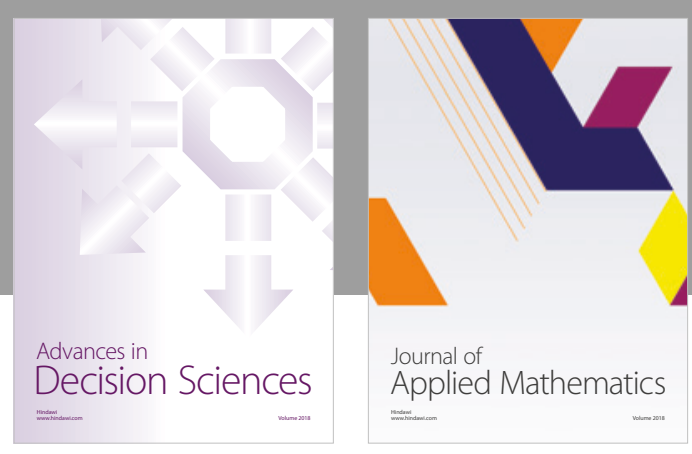

Journal of

Applied Mathematics
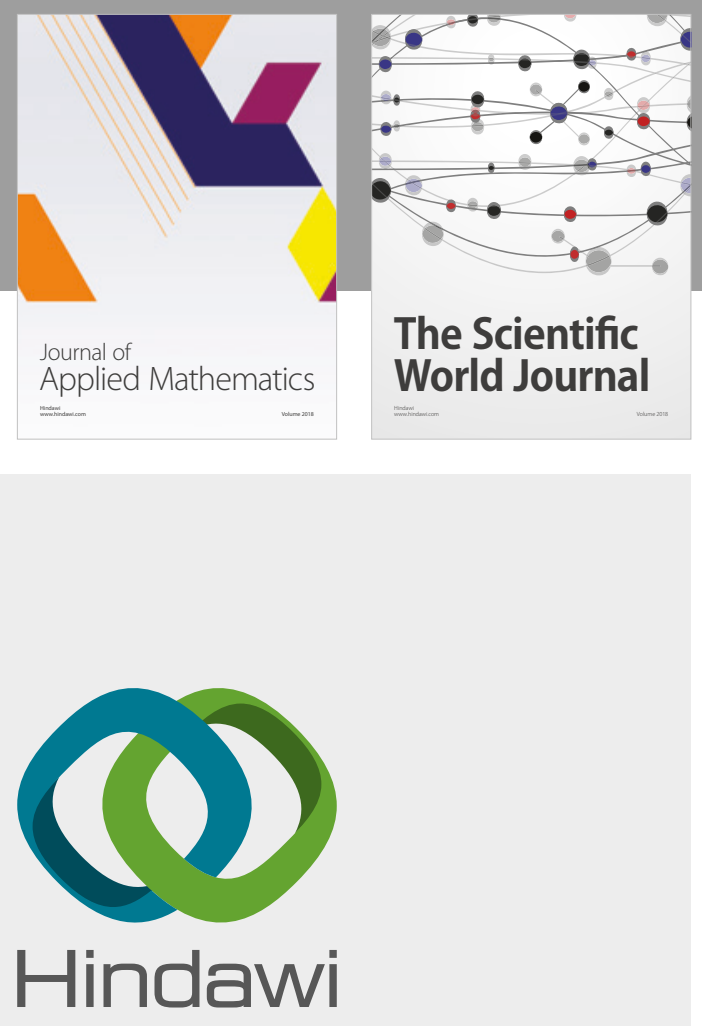

Submit your manuscripts at

www.hindawi.com

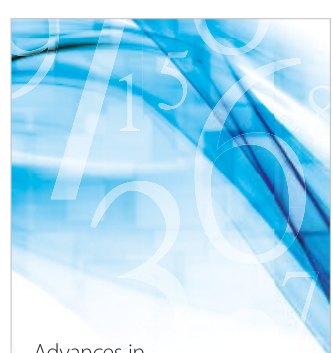

Advances in
Numerical Analysis
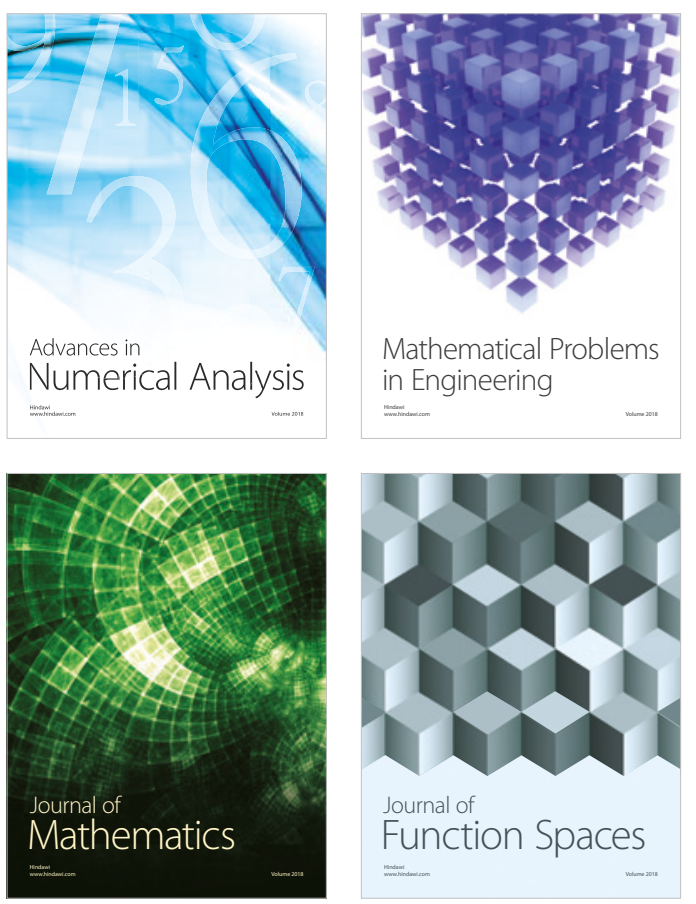

Mathematical Problems in Engineering

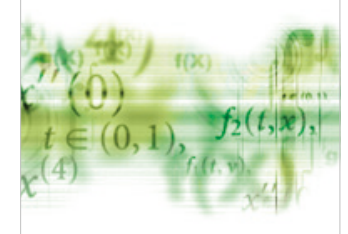

International Journal of

Differential Equations

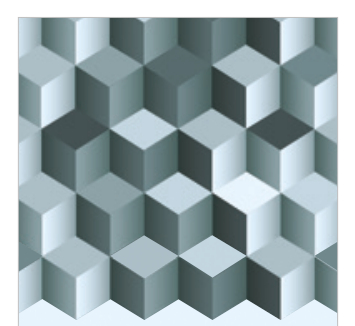

Journal of

Function Spaces

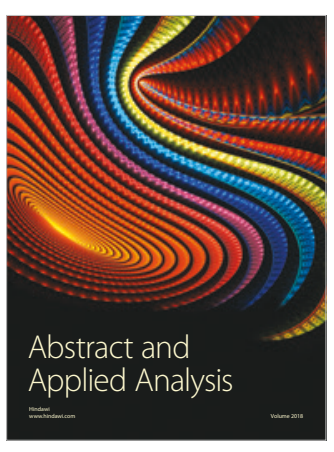

The Scientific

World Journal

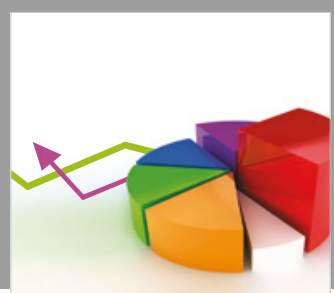

Journal of

Probability and Statistics
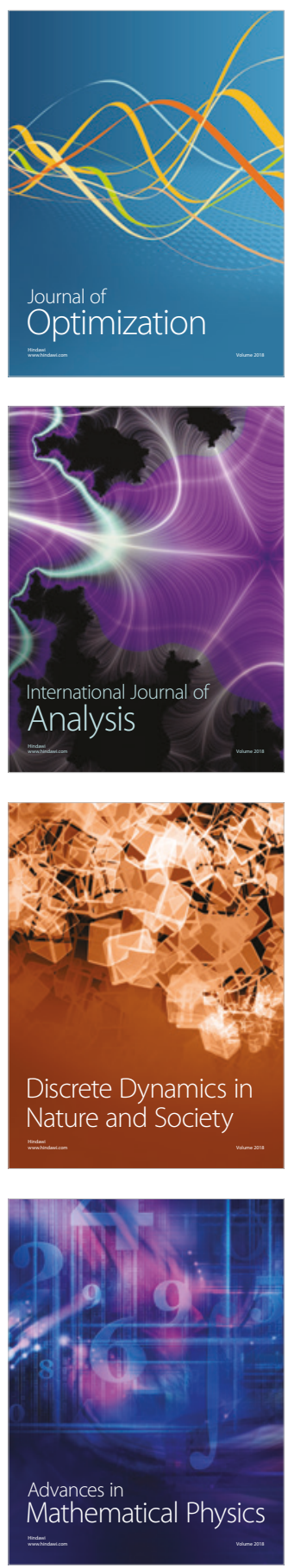